\title{
STAT3-PTTG11 abrogation inhibits proliferation and induces apoptosis in malignant glioma cells
}

\author{
LISHAN CUI ${ }^{1,2^{*}}$, LANXI XU $^{3^{*}}$, GUANLING WANG $^{3}$, JING WEN $^{3}$, LILI LUO $^{3}$, HAITAO ZHAO $^{1}$, \\ SHUIDE CHEN $^{1}$, MINGCHENG ZHENG ${ }^{1}$, CUILING SUN $^{4}$, XIN JIN $^{3}$ and LICHAO YANG ${ }^{3}$ \\ ${ }^{1}$ Department of Neurosurgery, Xiang'an Branch, The First Affiliated Hospital of Xiamen University; \\ ${ }^{2}$ Department of Neurosurgery, Xiamen Fifth Hospital, Xiamen, Fujian 361005; \\ ${ }^{3}$ Xiamen Key Laboratory of Chiral Drugs, School of Medicine; \\ ${ }^{4}$ School of Pharmacy, Xiamen University, Xiamen, Fujian 361102, P.R. China
}

Received November 27, 2019; Accepted May 13, 2020

DOI: 10.3892/ol.2020.11867

\begin{abstract}
Pituitary tumor transforming gene 1 (PTTG11) is abundantly expressed in glioma. Our previous study demonstrated that the downregulation of PTTG11 gene expression significantly inhibited the proliferation, migration and invasion ability, and increased the apoptosis of SHG44 glioma cells. However, the molecular mechanisms that regulate PTTG11 and its actions remain elusive. In the present study, CCK- 8 and flow cytometry assays were used to assess the proliferation/viability and apoptosis, respectively, of the human glioma U251 cell line. STAT3-PTTG1 signals were further evaluated by western blotting. The findings of the present study revealed that STAT3 induced PTTG11 expression, which subsequently induced downstream c-Myc and $\mathrm{Bcl}-2$ expression while inhibiting Bax expression, thereby promoting cell viability and inhibiting apoptosis. PTTG11 suppression via siRNA inhibited the viability and increased the apoptosis of glioma cells induced by the STAT3 activator S3I-201. c-Myc and Bcl-2 expression was suppressed by PTTG11 inhibition. The findings of the present study suggest that the STAT3-PTTG11 signaling pathway may play an important role in glioma progression by regulating cell proliferation and apoptosis.
\end{abstract}

\section{Introduction}

According to the World Health Organization report 2016, meningiomas are the most common primary tumors of the

Correspondence to: Dr Lichao Yang or Professor Xin Jin, Xiamen Key Laboratory of Chiral Drugs, School of Medicine, Xiamen University, Chengzhi Building, 4211 Xiang'an South Road, Xiamen, Fujian 361102, P.R. China

E-mail: yanglc116@xmu.edu.cn

E-mail: xinjin@xmu.edu.cn

${ }^{*}$ Contributed equally

Key words: malignant glioma, pituitary tumor transforming gene 1, STAT3, proliferation, apoptosis central nervous system in adults in the world (1). Glioma grows in an expansive and invasive manner, and tends to progress to a higher grade (2). Despite aggressive treatment (such as surgery followed by radiotherapy or chemotherapy), the median survival time for patients with GBM is only 14.6 months and most patients die within 2 years (3). The complexity of the genesis of malignant gliomas involves different genetic and molecular pathways (4). Epidermal growth factor receptor gene amplification and phosphatase and tensin homolog mutations are more common in primary GBM than secondary GBM. In secondary GBM, mutations occur more commonly in the isocitrate dehydrogenase 1 or 2 and TP53 genes $(5,6)$. In $~ 80 \%$ of GBMs, there are also changes in tyrosine kinase activity transmembrane receptor signaling pathways, the p53 pathway (TP53/mouse double minute 2 homolog/p14ARF), the phosphorylated retinoblastoma (RB) pathway [RB1/cyclin-dependant kinase (CDK) inhibitor 2A/CDK4] and the telomerase reverse transcriptase promoter region (pTERT) $(7,8)$. The high variation in the genes involved in GBM is an important reason for the poor efficacy of chemotherapy drugs. Therefore, treatment of these highly aggressive tumors is quite challenging. The understanding of the various important genes involved in glioma and the underlying signaling pathways activated during the process of carcinogenesis will reveal the nature of glioma development and provide new insight into the treatment of glioma.

Human pituitary tumor-transforming gene 1 (PTTG11) is a multifunctional proto-oncogene that is upregulated in various tumors, including glioma and hepatocellular carcinoma (9). The upregulation of PTTG11 is associated with tumor invasion, progression and angiogenesis, suggesting that PTTG1 may play a crucial role in tumorigenesis (10). PTTG1 has been identified as a key 'signature gene', with high levels of expression predicting metastasis in multiple tumor types, such as breast, prostate and ovarian cancer (11). Our previous study demonstrated that the downregulation of PTTG11 gene expression significantly inhibited the proliferation, migration and invasion ability, and increased the apoptosis of SHG44 glioma cells (12). These studies suggest that PTTG1 is a potential oncogene involved in tumor development, invasion and angiogenesis. However, the molecular mechanisms involved in the regulation of PTTG1 and its actions remain elusive. 
Signal transducer and activator of transcription 3 (STAT3) is an important regulatory factor that modulates tumor cell proliferation, apoptosis, invasion and metastasis (13). Several previous studies have demonstrated that STAT3 signaling plays an important role in the growth of gliomas, and increased STAT3 activation has been associated with the progression of pathological stages and worse overall survival (14-16). S3I-201 is a novel and selective STAT3 inhibitor of the Stat3/Stat3 complex, STAT3 tyrosine phosphorylation and DNA binding, exerting antitumor properties. Furthermore, the interleukin (IL)-6/JAK/STAT3 pathway is involved in the pathogenesis of numerous human malignancies $(17,18)$. In cancer, increased IL-6 levels result in hyperactivation of JAK/STAT3 signaling, which is typically associated with a poorer prognosis (19). In the process of tumorigenesis and development, PTTG11 and STAT3 can affect the regulation of the cell cycle and participate in biological processes, such as cell apoptosis and proliferation. PTTG11 and STAT3 regulate some mutual downstream target genes, including c-Myc and Bax/Bcl-2 (20,21). Overall, the PTTG11 pathway may be involved in STAT3 modulated tumor cell proliferation and apoptosis, although additional studies are required to confirm this hypothesis.

Our previous study demonstrated that the downregulation of PTTG11 gene expression significantly inhibited the proliferation, migration and invasion ability, and increased the apoptosis of SHG44 glioma cells. However, the molecular mechanisms that regulate PTTG11 and its actions remain elusive. In the present study, CCK-8 and flow cytometry assays were used to assess the proliferation/viability and apoptosis, respectively, of the human glioma U251 cell line. The purpose of this study is to explore the effect of PTTG1 on the proliferation and apoptosis of glioma cell U251 and explore its mechanism.

\section{Materials and methods}

Materials. siRNA-PTTG11, scrambled negative control siRNA (siRNA-NC) and riboFECT CP Transfection kit were purchased from Guangzhou RiboBio Co., Ltd (https://www. ribobio.com/). Cell Counting Kit-8 (CCK-8) was purchased from Dalian Meilun Biotechnology Co., Ltd. An inhibitor (S3I-201) and agonist (IL-6) of STAT3 were purchased from Sigma-Aldrich; Merck KGaA. Based on previous reports, a dose of $200 \mu \mathrm{m} \mathrm{S3I-201} \mathrm{was} \mathrm{used} \mathrm{in} \mathrm{the} \mathrm{present} \mathrm{study} \mathrm{(21-23).}$ The annexin V-FITC/propidium iodide (PI) apoptosis kit was purchased from Nanjing KeyGen Biotech Co., Ltd. The primary antibodies against PTTG11 (cat. no. ab79546) and Bax (cat. no. ab32503) were purchased from Abcam. The antibodies against c-myc (cat. no. D84C12), p-STAT3 (cat. no. D3A7) and STAT3 (cat. no. 124H6) were purchased from Cell Signaling Technology, Inc. The primary antibody against $\beta$-actin (cat. no. 20536-1-AP) was purchased from the Proteintech Group, Inc. The secondary antibodies, horse radish peroxidase (HRP)-labeled goat anti-rabbit IgG (cat. no. GAR007) and HRP-labeled goat anti-mouse IgG (cat. no. GAM007) were purchased from Hangzhou Multi Sciences (Lianke) Biotech. Co., Ltd.

Cell culture. U251 cells were cultured in Dulbecco's Modified Eagle's Medium (DMEM) (Hyclone; GE Healthcare Life Sciences) containing $10 \%$ fetal bovine serum (Thermo Fisher
Scientific Inc.) at $37^{\circ} \mathrm{C}$ in $5 \% \mathrm{CO}_{2}$. The medium was replaced at 48-h intervals. After the cells had reached $80-90 \%$ confluency, the cells were passaged.

Cell transfection. U251 cells in the logarithmic growth phase were selected for transfection. The cells were divided into 3 groups: A blank group (non-transfected cells), a negative control group (cells transfected with $50 \mathrm{nM}$ siRNA-negative control) and an siRNA-PTTG11 group (cells transfected with siRNA-PTTG11). According to our previous study (12), the sequence of the siRNA-PTTG11 was 5'-GGGAGATCTCAG TTTCAA-3' and it was used at a concentration of $50 \mathrm{nM}$. The cells were placed in 6-well plates at a density of $1 \times 10^{6}$ cells/well. When their confluence reached $70 \%$, cell transfection was performed according to the manufacturer's instructions from the transfection kit. After $24 \mathrm{~h}$, the medium was replaced with fresh medium in which siRNA-NC and siRNA-PTTG11 were added along with $80 \mathrm{ng} / \mathrm{ml} \mathrm{IL-6}$ for $24 \mathrm{~h}$.

CCK-8 assay. To assess the proliferation of the U251 cells after different treatments, 5,000 cells/well were inoculated into 96-well plates and cultured for $24 \mathrm{~h}$. The cells were treated with different concentrations of S3I-201 $(0,50,100$ or $200 \mu \mathrm{M})$ or IL-6 $(20,40$ or $80 \mathrm{ng} / \mathrm{ml})$ for $0,6,12,24,48$ or $72 \mathrm{~h}$. The $0 \mu \mathrm{M}$ was used as the control. Next, $10 \mu \mathrm{l}$ of a CCK-8 solution was added to each well and the cells were incubated at $37^{\circ} \mathrm{C}$ for $1 \mathrm{~h}$, according to the manufacturer's protocols. Subsequently, the absorbance at $450 \mathrm{~nm}$ of each group was measured. The cell viability was calculated using the formula given in the manufacturer's instructions from the kit.

Flow cytometry. Transfected U251 cells were digested with $0.25 \%$ trypsinase, washed twice with $300 \mathrm{ml}$ prechilled PBS and then collected by centrifugation $800 \mathrm{x}$ g for $5 \mathrm{~min}$ at $4^{\circ} \mathrm{C}$. The cells were resuspended at $1-5 \times 10^{5}$ cells $/ \mathrm{ml}$ in $100 \mu \mathrm{l}$ binding buffer (eBioscience; Thermo Fisher Scientific, Inc.) followed by the addition of $5 \mu$ l Annexin V labeled with FITC and $10 \mu \mathrm{PI}$ in the dark at room temperature. After $15 \mathrm{~min}$, $400 \mu \mathrm{l}$ additional binding buffer was added to the reaction system. Cell apoptosis was analyzed using a flow cytometer (Attune NxT, Thermo Fisher Scientific, Inc.) at an excitation wavelength of $488 \mathrm{~nm}$. FlowJo version 10.5 (BD Biosciences) was used for analysis.

Western blotting. Human glioma U251 cells were lysed with RIPA buffer (Beijing Solarbio Science \& Technology Co., Ltd.) that contained protease inhibitors. After quantification with a BCA protein assay kit (Pierce; Thermo Fisher Scientific, Inc.) using a Multifunctional microplate reader (Molecular Devices). Total protein ( $20 \mu \mathrm{g} /$ lane) was separated on a $12 \%$ gel using sodium dodecyl sulfate polyacrylamide gel electrophoresis. Next, the proteins were transferred to polyvinylidene difluoride membranes. The 5\% Nonfat-Dried milk (Solarbio, Beijing, China) in TBST was used to block PVDF membranes at room temperature for $1 \mathrm{~h}$ followed by incubation with primary antibodies against PTTG11 (1:5,000), Bax (1:1,000), c-Myc $(1: 1,000)$, p-STAT3 $(1: 2,000)$, STAT3 $(1: 1,000)$, or $\beta$-actin $(1: 5,000)$ overnight at $4^{\circ} \mathrm{C}$. The membranes were then incubated with the the secondary antibodies (both 1:10,000) 
A

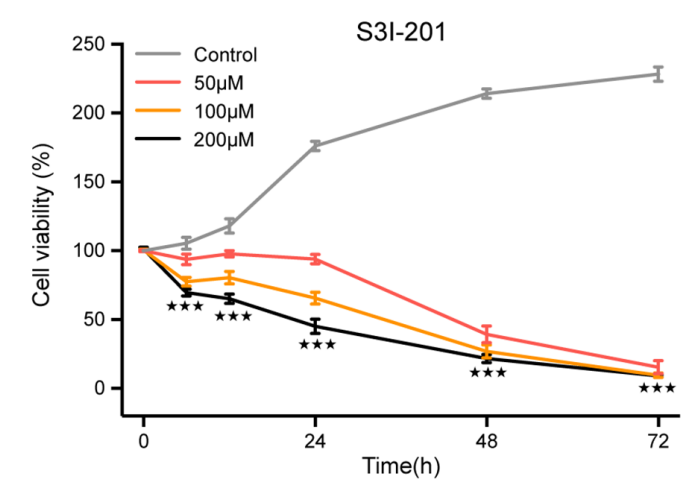

B

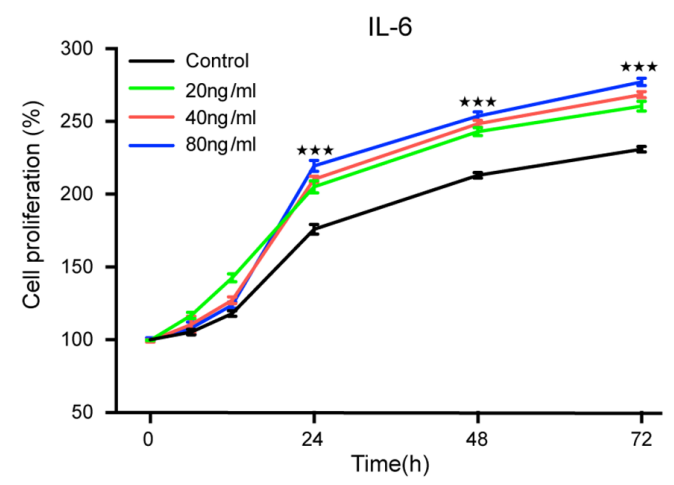

C
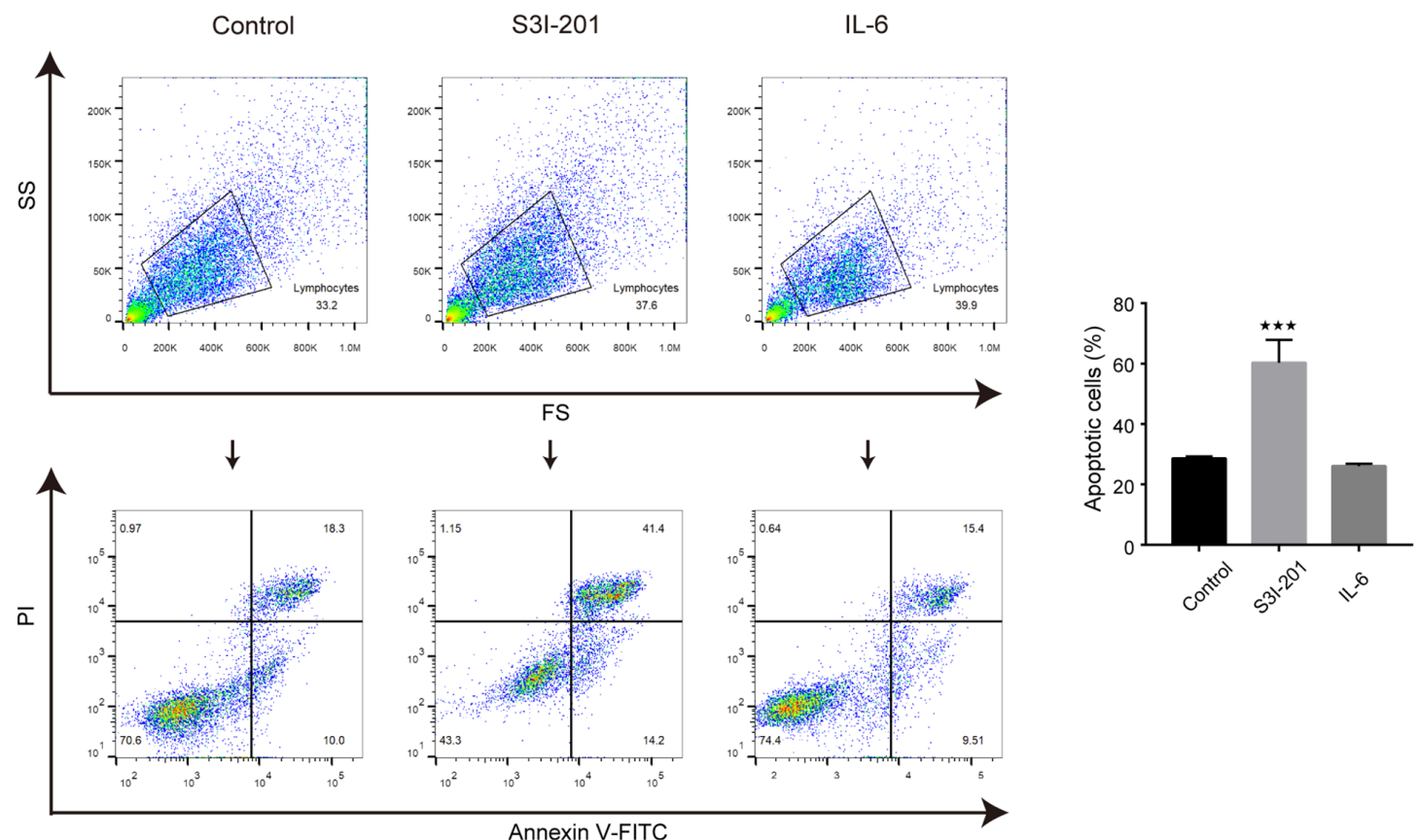

Figure 1. Effects of S3I-201 and IL-6 on proliferation and apoptosis of U251 cells. (A) Viability of U251 cells in the S3I-201 group was significantly decreased. (B) The viability of U251 cells in the IL-6 group was significantly increased. (C) S3I-201 could promote the apoptosis of U251 cells while IL-6 inhibited apoptosis. The experiment was repeated three times. ${ }^{* * *} \mathrm{P}<0.001$ compared with the control group (set to $100 \%$ ). IL, interleukin; SS, side scatter; FS, forward scatter; PI, propidium iodide.

at room temperature for $1 \mathrm{~h}$. ECL solution (EMD Millipore) was used to detect the proteins on the membrane. The relative protein levels were normalized to $\beta$-actin.

Statistical analysis. Experimental result data are expressed as the mean \pm SEM of three separate experiments. Data for cell viability were compared using two-way ANOVA followed by Bonferroni's post hoc test, and one-way ANOVA with Tukey's post hoc test for the remainder of the multiple comparisons performed. The analyses were performed using Prism 7 for Windows (GraphPad Software Inc.). $\mathrm{P}<0.05$ was considered to indicate a statistically significant difference.

\section{Results}

Effects of S3I-201 and IL-6 on viability and apoptosis of U251 cells. It has been reported that S3I-201 and IL-6 are an inhibitor and an agonist of STAT3, respectively $(22,23)$. To investigate the effects of IL-6 and S3I-201 on glioma cells, the CCK-8 assay was used at $0,6,12,24,48$ and $72 \mathrm{~h}$ after treatment with different doses of S3I-201 $(0,50,100$ or $200 \mu \mathrm{M})$ or IL-6 $(0,20,40$ or $80 \mathrm{ng} / \mathrm{ml})$ to measure U251 cell viability and proliferation, respectively. The results demonstrated that the viability of U251 cells treated with S3I-201 at dosage of $200 \mu \mathrm{M}$ was significantly decreased compared with that of the control group (Fig. 1A). When U251 cells were treated with IL-6 at dosage of $80 \mathrm{ng} / \mathrm{ml}$, the proliferation of the U251 cells was significantly increased compared with that in the control group (Fig. 1B).

In addition, flow cytometry was performed to confirm that IL-6 $(80 \mathrm{ng} / \mathrm{ml})$ and S3I-201 $(200 \mu \mathrm{M})$ affected apoptosis of the $\mathrm{U} 251$ cells. The results revealed that the percentage of apoptotic cells was significantly increased in the S3I-201 group compared with that in the control group (Fig. 1C). However, the percentage 

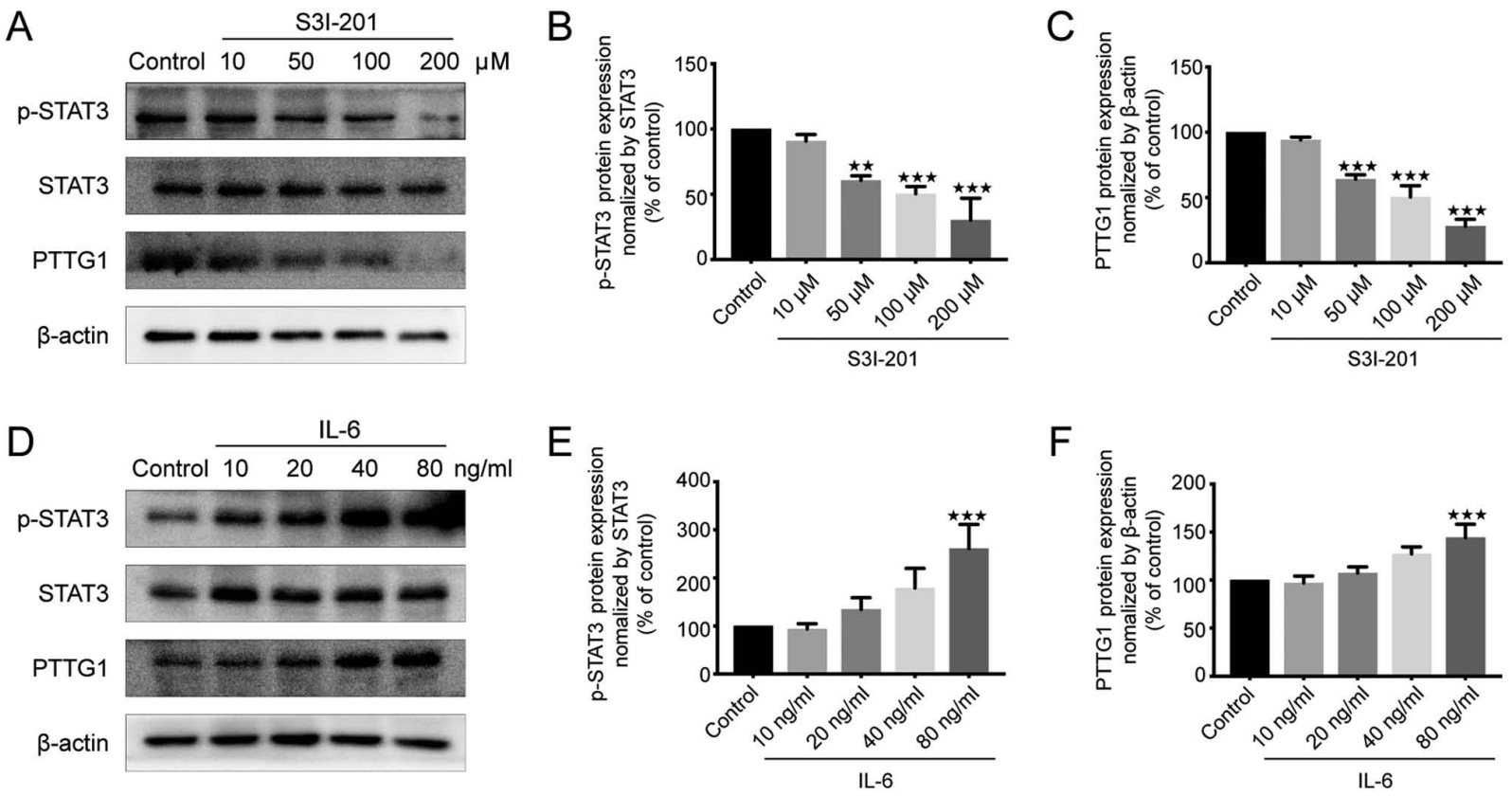

Figure 2. Effect of different doses of S3I-201 and IL-6 on the protein expression of p-STAT3 and PTTG11 proteins in U251 cells. (A) Western blotting results of p-STAT3, STAT3 and PTTG11 proteins in U251 cells treated with S3I-201. Expression levels of the (B) p-STAT3 and (C) PTTG11 proteins were both decreased after S3I-201 treatment. (D) Western blotting results of p-STAT3, STAT3 and PTTG11 proteins in U251 cells treated with IL-6. Expression levels of (E) p-STAT3 and (F) PTTG11 proteins were both increased after IL-6 treatment. Quantified results were normalized to $\beta$-actin expression. Values were expressed as percentages compared to control group (set to $100 \%$ ). The experiment was repeated three times. ${ }^{* * *} \mathrm{P}<0.01,{ }^{* * * *} \mathrm{P}<0.001$ compared with the control group (set to 100\%). IL, interleukin; p, phosphorylated; PTTG11, pituitary tumor transforming gene 1.

of apoptotic cells was decreased in the IL-6 group compared with the control group, although this did not reach statistical significance (Fig. 1C). In conclusion, these data implied that S3I-201 could decrease U251 cell viability and promote apoptosis, while IL-6 increased proliferation and had no effect on apoptosis. Thus, it was hypothesized that STAT3 could promote the viability of and inhibit the apoptosis of U251 cells.

Effects of S3I-201 and IL-6 on the protein expression of p-STAT3 and PTTG11 in U251 cells. PTTG11 is known to promote the proliferation of glioma cells (24). To elucidate the association between STAT3 and PTTG11, western blotting was used to measure the protein expression levels of p-STAT3 and PTTG11. The results demonstrated that the expression levels of p-STAT3 and PTTG11 were significantly decreased in the 50,100 and $200 \mu \mathrm{M} \mathrm{S} 3 \mathrm{I}-201$ groups compared with those in the control group (Fig. 2A-C). However, there was no statistically significant difference between the $10 \mu \mathrm{M} \mathrm{S} 3 \mathrm{I}-201$ and control groups (Fig. 2A-C). By contrast, the p-STAT3 and PTTG11 levels were increased in the IL-6 group compared with those in the control group, with this effect reaching significance when cells were treated with $80 \mathrm{ng} / \mathrm{ml}$ IL-6 (Fig. 2D-F). The changes in PTTG11 and p-STAT3 demonstrated similar trends to each other when treated with S3I-201 and IL-6. Therefore, it was concluded that the protein expression of the p-STAT3 protein was positively associated with PTTG11 protein expression.

IL-6 promotes the proliferation of U251 cells by affecting the expression of PTTG11. The aforementioned results demonstrated that IL- 6 promoted the proliferation of U251 cells and increased the expression of PTTG11 in U251 cells. To verify whether IL- 6 promotes the proliferation of U251 by upregulating the expression of PTTG11, U251 cells were transfected with siRNA-PTTG11 and treated with IL-6, and the cell viability and apoptotic rates of U251 cells were investigated. The results of the CCK- 8 assays revealed that the difference in U251 cell viability between the control and siRNA-NC group was not statistically significant (Fig. 3A). Compared with the siRNA-NC group, the viability of the siRNA-PTTG11 and siRNA-PTTG11+IL-6 groups was significantly decreased, while for the siRNA-NC+IL- 6 group it was significantly increased between 24 and $72 \mathrm{~h}$ (Fig. 3A). There was no statistically significant difference between the siRNA-PTTG11 and siRNA-PTTG11+IL-6 groups (Fig. 3A).

Flow cytometry experiments were performed to further verify these results. The results demonstrated that compared with that in the siRNA-NC group, the percentage of apoptotic cells was significantly increased in the siRNA-PTTG11 and siRNA-PTTG11+IL-6 groups; however, it was decreased in the siRNA-NC+IL-6 group (Fig. 3B and C). There was no statistically significant difference in the apoptosis rate between the siRNA-PTTG11+IL-6 group and the siRNA-PTTG11 group (Fig. 3B and C). These results suggest that silencing PPTG1 could affect cell proliferation and apoptosis, but that IL-6 could not effectively weaken this effect. In conclusion, IL-6 regulates the proliferation and apoptosis of U251 cells by affecting the expression of PTTG11.

IL-6 affects U251 apoptosis and cell viability by altering the expression of STAT3-PTTG11-related proteins. The aforementioned results demonstrated that the expression levels of p-STAT3 and PTTG11 were positively associated in U251 cells, while IL-6, as an agonist of STAT3, could upregulate the expression of PTTG11 to promote cell proliferation. It was therefore 
A

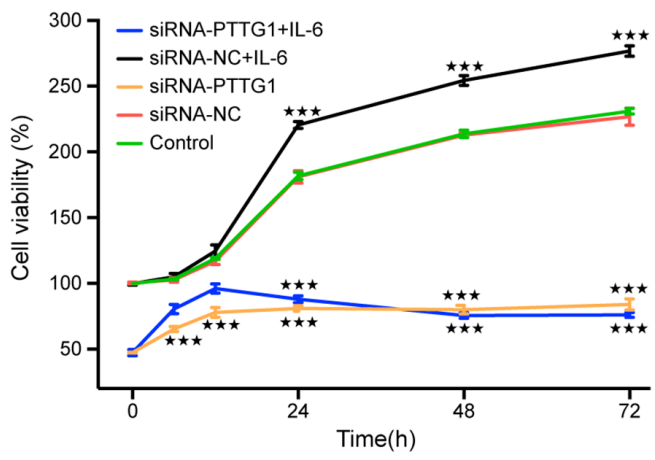

$\mathrm{B}$

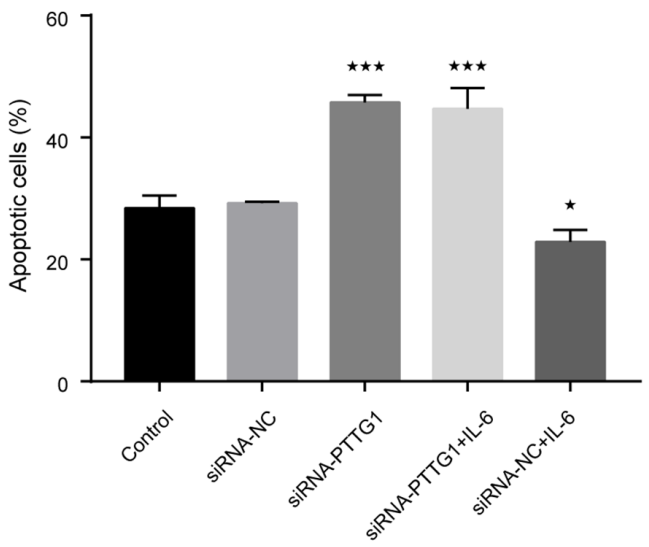

C

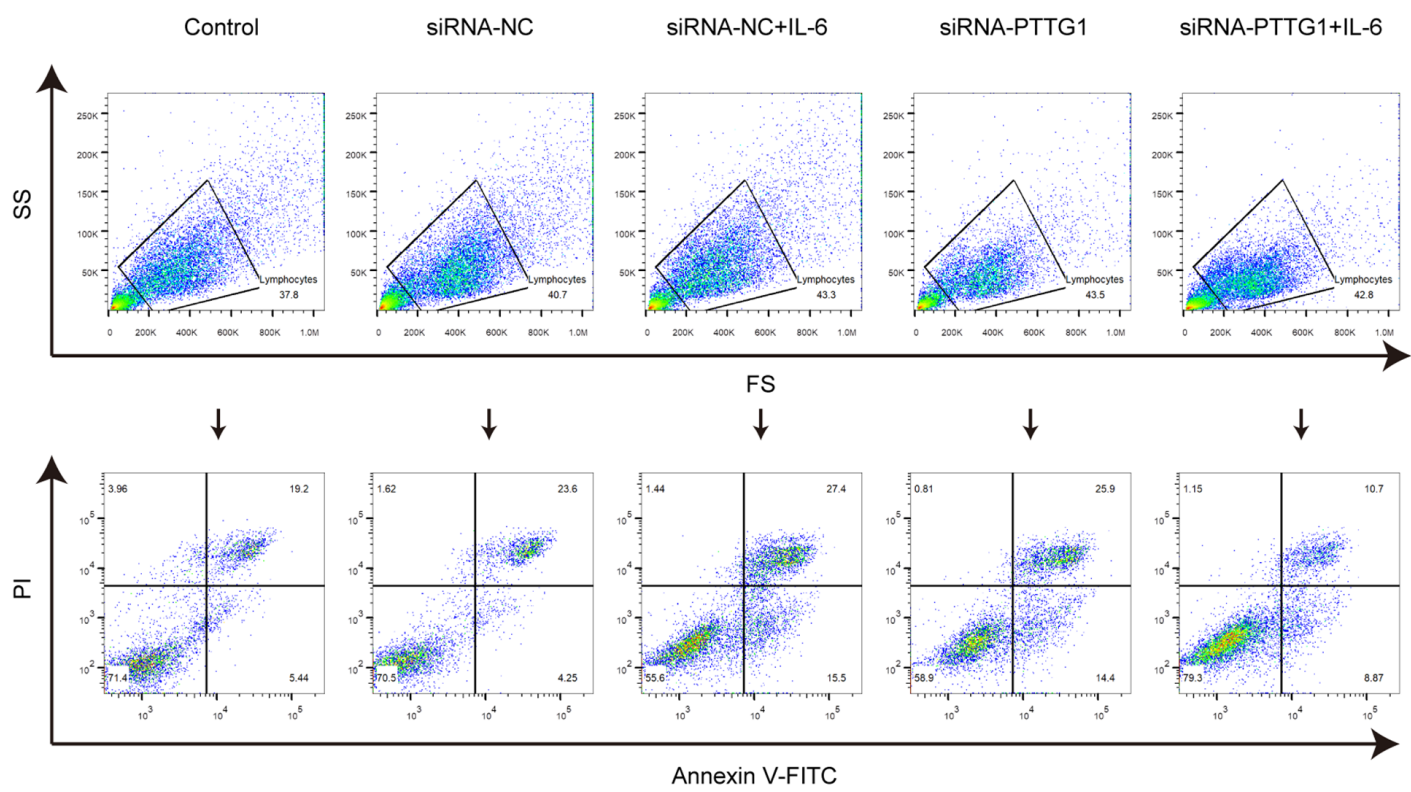

Figure 3. Effect of siRNA-PTTG11 on the viability and apoptosis of U251 cells. The control group was untreated, and negative control group cells were treated with NC siRNA. (A) Viability of U251 cells in each group after cell transfection. The viability of the U 251 cells decreased after the cells were transfected with siRNA-PTTG11, and there was no statistically significant difference between the siRNA-PTTG11 and siRNA-PTTG11+IL-6 (80 ng/ml) groups after $24 \mathrm{~h}$, while the viability of the siRNA-NC+IL-6 group was significantly increased from 24 to $72 \mathrm{~h}$. (B and C) Apoptosis rate of U251 cells in each group after transfection. Apoptosis was significantly increased in the siRNA-PTTG11 and siRNA-PTTG11+IL-6 groups, but decreased in the siRNA-NC+IL-6 group compared with siRNA-NC group. ${ }^{*} \mathrm{P}<0.05$ and ${ }^{* * *} \mathrm{P}<0.001$ compared with the control group (set to $100 \%$ ). IL, interleukin; SS, side scatter; FS, forward scatter; PI, propidium iodide; PTTG11, pituitary tumor transforming gene 1; NC, negative control; si, small interfering.

hypothesized that STAT3 might act upstream of PTTG11 and regulate the apoptosis and proliferation of $\mathrm{U} 251$ cells by altering the expression of PTTG11. To prove this hypothesis, U251 cells were transfected with siRNA-PTTG11. Following confirmation of transfection efficiency, western blotting was performed to observe the changes in protein levels (Fig. 4A and C). The results indicated that silencing PTTG11 had no significant effect on p-STAT3 (Fig. 4A and B). However, IL-6 could upregulate the expression of p-STAT3 in U251 cells (Fig. 4B). The expression of PTTG11 was increased in the siRNA-NC+IL-6 group; however, IL-6 did not obviously increase the level of PTTG11 after silencing of PTTG11 (Fig. 4C). In addition, proliferation-associated proteins (c-Myc and $\mathrm{Bcl}-2)$ (25) were decreased in the siRNA-PTTG11 and siRNA-PTTG11+IL-6 groups compared with the levels in the siRNA-NC group (Fig. 4D and E). By contrast, the expression of c-Myc and Bcl-2 was enhanced in the siRNA-NC-IL-6 group compared with the siRNA-NC group (Fig. 4D and E). In addition, the levels of the apoptosis-associated protein (Bax) (26) in the siRNA-PTTG11 and siRNA-PTTG11+IL-6 groups were significantly higher compared with the level in the siRNA-NC group (Fig. 4F). In the siRNA-NC+IL- 6 group, there was a decrease in the level of Bax protein compared with the siRNA-NC group (Fig. 4F). In conclusion, IL-6 could upregulate the expression of PTTG11, c-Myc and Bcl-2, and decrease the expression of BAX by inducing the expression of p-STAT3, which could be achieved by regulating the target gene PTTG11.

\section{Discussion}

Due to the failure of conventional therapeutic strategies (surgery combined with radiotherapy and chemotherapy) (27,28), 

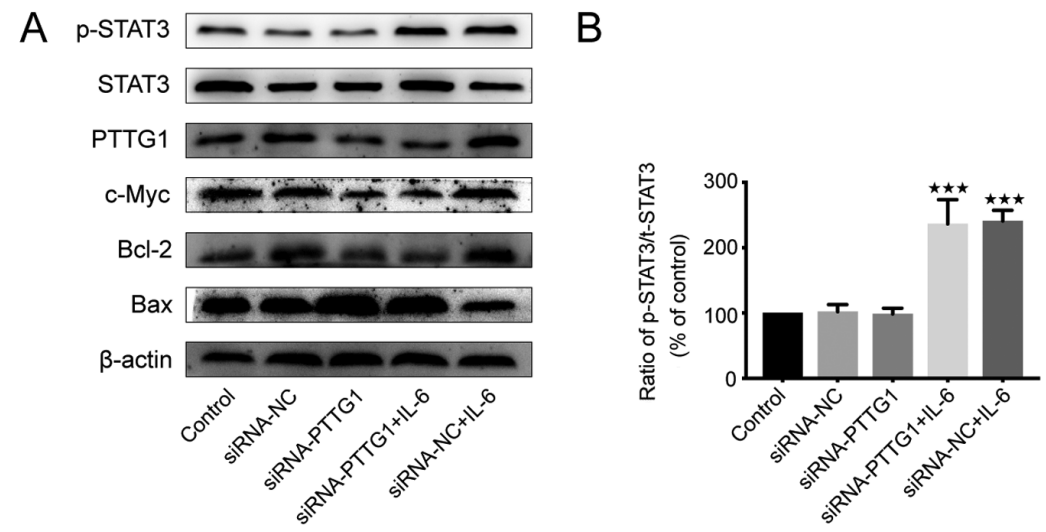

C
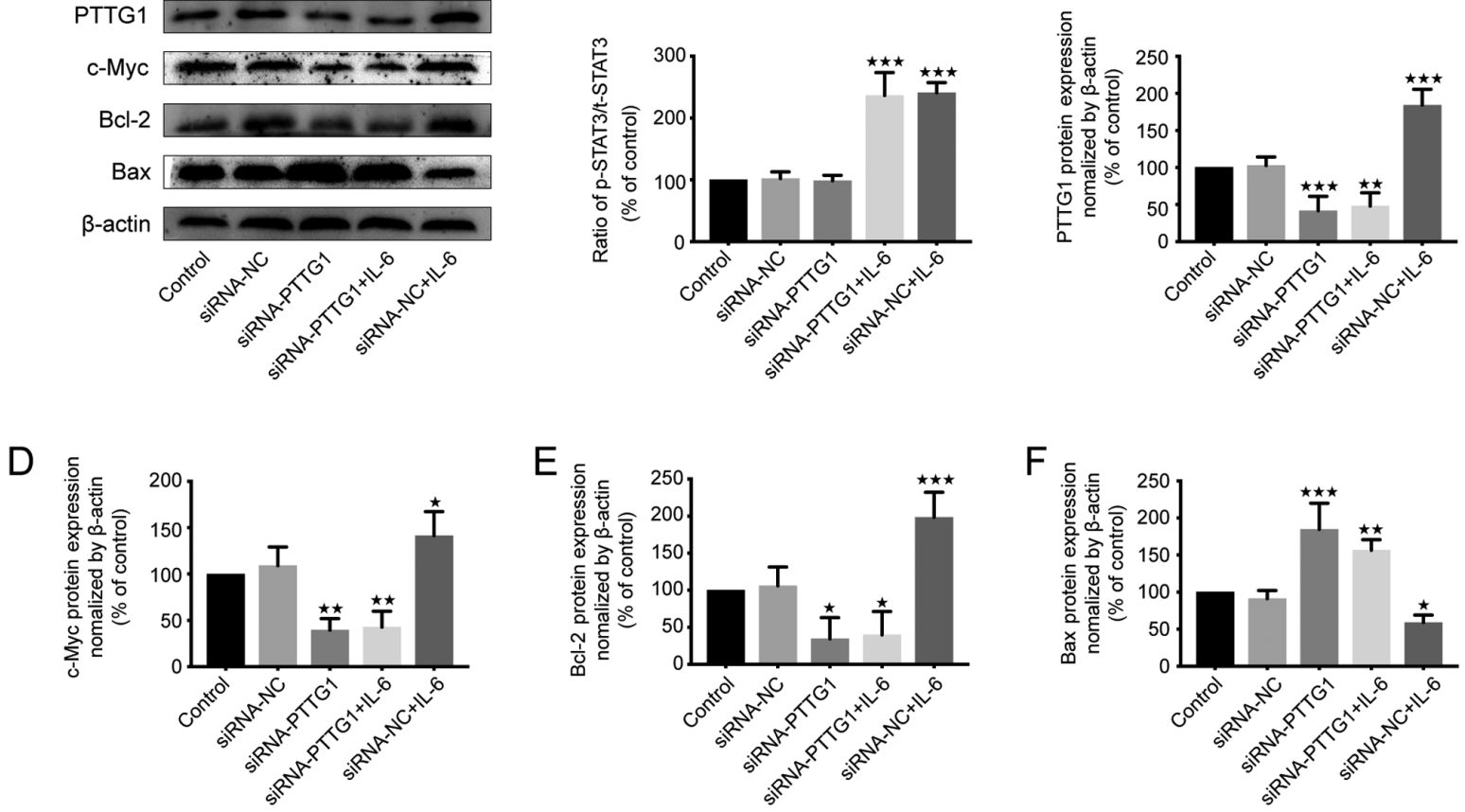

Figure 4. IL-6 affects apoptosis and proliferation by altering the expression of related proteins in U251 cells. The control group was untreated, and negative control group cells were treated with NC siRNA. (A) Expression of proliferation and apoptosis-related proteins in U251 cells of each group as shown by western blotting. The expression levels of p-STAT3, PTTG11, c-Myc, Bcl-2 and Bax in U251 cells after the indicated treatment. (B) Whether PTTG11 was silenced or not, IL-6 could upregulate the expression of p-STAT3. (C) IL-6 at dosage of $80 \mathrm{ng} / \mathrm{ml}$ could upregulate the expression of PTTG11. This effect was not obvious when PTTG11 was silenced. (D) c-Myc and (E) Bcl-2 were decreased in the siRNA-PTTG11 and siRNA-PTTG11+IL-6 groups, but enhanced in the siRNA-NC+IL-6 group compared with siRNA-NC group. (F) Bax levels in the siRNA-PTTG11 and siRNA-PTTG11+IL-6 groups were notably higher but were at their lowest in the siRNA-NC+IL-6 group compared with siRNA-NC group. ${ }^{*} \mathrm{P}<0.05,{ }^{* *} \mathrm{P}<0.01$ and ${ }^{* * *} \mathrm{P}<0.001$ compared with the control group (set to $100 \%$ ). IL, interleukin; PTTG11, pituitary tumor transforming gene 1; NC, negative control; si, small interfering.

an understanding of the molecular mechanisms of glioma will be helpful for treating the disease and for delivering tumoricidal agents. The oncogenic transcription factor STAT3 is a key signaling hub, regulating a number of tumor-related processes, including proliferation, migration, apoptosis-resistance, angiogenesis and immune evasion (29). A previous study demonstrated that PTTG11 plays an important role in gene modulation, angiogenesis, mitoses, cell cycle control, cell transformation, DNA repair and cell apoptosis (30). A high expression level of PTTG11 has been observed in U251 glioma cells. Both STAT3 and PTTG11 have been implicated in multiple steps of glioma development and progression $(19,24)$, but the mechanisms that regulate their coordinated functions were not known.

The findings of the present study used an activator and inhibitor of STAT3 to reveal that a positive association exists between the extent of STAT3 and PTTG11 activation. The present study demonstrated that both p-STAT3 and PTTG11 protein levels were significantly decreased in the S3I-201 group compared with those in the NC group (Fig. 2A-C). However, p-STAT3 and PTTG11 protein levels increased significantly in the IL-6 group compared with those in the NC group (Fig. 2D-F). When PTTG1-siRNA was used, the effects of STAT3 on proliferation were significantly suppressed and PTTG1 silencing increased the apoptosis of U251 cells. These results demonstrated that PTTG1 may be one of the genes targeted by the STAT3 signaling pathway that acts to promote glioma proliferation and apoptosis.
In addition, in the present study, the protein expression of genes downstream of the STAT3-PTTG1 signaling pathway was assessed. Therefore, the effect of PTTG1-siRNA transfection on IL-6-induced proliferation and apoptosis-related protein expression in U251 cells was investigated. Following PTTG1-siRNA transfection, the protein expression levels of PTTG11, c-Myc and Bcl-2 were decreased, while that of Bax was increased. IL-6-induced STAT3 activation upregulated PTTG11, c-Myc and Bcl-2 protein expression, and downregulated Bax protein expression, which was achieved by regulating the target gene PTTG11. The findings of the present study demonstrated that c-Myc, which is responsible for the inhibition of cell proliferation, was downregulated after PTTG11-siRNA transfection. It was also demonstrated that Bcl-2 was downregulated and BAX was upregulated following PTTG11-siRNA transfection, showing that together, these processes are responsible for increased levels of cell apoptosis. These results are similar to previous reports $(24,25)$.

Of course, this study also has limitations. We used the U251 cell line to study the role of PTTG1 in glioma proliferation and apoptosis, and explored its mechanism. Although U251 is a widely used and recognized cell for gliomas study, but the use of single cell line still makes this research slightly underweight. In conclusion, the findings of the present study demonstrated that PTTG11 is a downstream gene of STAT3. STAT3 induces PTTG11 expression, which then induces $\mathrm{c}-\mathrm{Myc}$ and $\mathrm{Bcl}-2$ expression, and inhibits BAX expression, 
thereby promoting cell proliferation and inhibiting apoptosis The findings of the present study suggest that PTTG11 plays an important role in glioma progression by regulating cell proliferation and apoptosis. PTTG11 may be a potential therapeutic target for blocking glioma cell proliferation.

\section{Acknowledgements}

Not applicable.

\section{Funding}

This study was supported by grants from the Fundamental Research Funds for the Central Universities (grant no. 20720180042), the Health Science Research Personnel Training Program of Fujian Province (grant no. 2016-CXB-12) and the Natural Science Foundation of Fujian, China (grant no. 2016D019).

\section{Availability of data and materials}

All data used and/or analyzed during the present study are available from the corresponding author on reasonable request.

\section{Authors' contributions}

LC, XJ and LY designed the experiments and wrote the manuscript. LX, GW, JW and LL were involved in the design of the study and performed the experiments. HZ, SC, MZ and CS performed the statistical analysis. All authors read and approved the final manuscript.

\section{Ethics approval and consent to participate}

Not applicable.

\section{Patient consent for publication}

Not applicable.

\section{Competing interests}

The authors declare that they have no competing interests.

\section{References}

1. Louis DN, Perry A, Reifenberger G, von Deimling A, Branger DF, Cavenee WK, Ohgaki H, Wiestler OD, Kleihues P and Ellison DW: The 2016 world health organization classification of tumors of the central nervous system: A summary. Acta Neuropathol 131: 803-820, 2016.

2. Hawkins-Daarud A, Rockne RC, Anderson AR and Swanson KR Modeling tumor-associated edema in gliomas during anti-angiogenic therapy and its impact on imageable tumor. Front Oncol 3: 66, 2013

3. Lin L, Cai J and Jiang C: Recent advances in targeted therapy for glioma. Curr Med Chem 24: 1365-1381, 2017.

4. Omuro A and DeAngelis LM: Glioblastoma and other malignant gliomas: A clinical review. JAMA 310: 1842-1850, 2013.

5. Navarro L, Gil-Benso R, Megías J, Muñoz-Hidalgo L, SanMiguel T, Callaghan RC, González-Darder JM, López-Ginés C and Cerdá-Nicolás MJ: Alteration of major vault protein in human glioblastoma and its relation with EGFR and PTEN status. Neuroscience 297: 243-251, 2015.
6. Furnari FB, Fenton T, Bachoo RM, Mukasa A, Stommel JM, Stegh A, Hahn WC, Ligon KL, Louis DN, Brennan C, et al: Malignant astrocytic glioma: Genetics, biology, and paths to treatment. Genes Dev 21: 2683-2710, 2007.

7. Inda MM, Bonavia R, Mukasa A, Narita Y, Sah DW, Vandenberg S, Brennan C, Johns TG, Bachoo R, Hadwiger P, et al: Tumor heterogeneity is an active process maintained by a mutant EGFR-induced cytokine circuit in glioblastoma. Genes Dev 24: 1731-1745, 2010.

8. Muñoz-Hidalgo L, San-Miguel T, Megías J, Monleón D, Navarro L, Roldán P, Cerdá-Nicolás $M$ and López-Ginés C: Somatic copy number alterations are associated with EGFR amplification and shortened survival in patients with primary glioblastoma. Neoplasia 22: 10-21, 2020.

9. Benito R, Gil-Benso R, Quilis V, Perez M, Gregori-Romero M, Roldan P, Gonzalez-Darder J, Cerdá-Nicolas $M$ and Lopez-Gines C: Primary glioblastomas with and without EGFR amplification: Relationship to genetic alterations and clinicopathological features. Neuropathology 30: 392-400, 2010.

10. Akizuki K, Sekine M, Kogure Y, Kameda T, Shide K, Koya J, Kamiunten A, Kubuki Y, Tahira Y, Hidaka T, et al: TP53 and PTEN mutations were shared in concurrent germ cell tumor and acute megakaryoblastic leukemia. BMC Cancer 20: 5, 2020.

11. Solbach C, Roller M, Fellbaum C, Nicoletti M and Kaufmann M: PTTG1 mRNA expression in primary breast cancer: A prognostic marker for lymph node invasion and tumor recurrence. Breast 13: 80-81, 2004.

12. Li Y, Zhou LP, Ma P, Sui CG, Meng FD, Tian X, Fu LY and Jiang YH: Relationship of PTTG1 expression with tumor invasiveness and microvessel density of pituitary adenomas: A meta-analysis. Genet Test Mol Biomarkers 18: 279-285, 2014.

13. Ramaswamy S, Ross KN, Lander ES and Golub TR: A molecular signature of metastasis in primary solid tumors. Nat Genet 33: 49-54, 2003.

14. Cui LS, Lin T, Xu LX, Wang GL, Lin JB, Feng SP, Cao Y, Cao Y, Song ZM and Jin X: The effect of down-regulated gene PTTG11 on proliferation, apoptosis, migration and invasion of human glioma cell SHG44. China Oncol 29: 338-344, 2019.

15. Johnston PA and Grandis JR: STAT3 signaling: Anticancer strategies and challenges. Mol Interv 11: 18-26, 2011.

16. Tu Y, Zhong Y, Fu J, Cao Y, Fu G, Tian X and Wang B: Activation of JAK/STAT signal pathway predicts poor prognosis of patients with gliomas. Med Oncol 28: 15-23, 2011.

17. Tan MSY, Sandanaraj E, Chong YK, Lim SW, Koh LW, Ng WH, Tan NS, Tan P, Ang BT and Tang C: A STAT3-based gene signature stratifies glioma patients for targeted therapy. Nat Commun 10: 3601, 2019.

18. Ouedraogo ZG, Biau J, Kemeny JL, Morel L, Verrelle P and Chautard E: Role of STAT3 in genesis and progression of human malignant gliomas. Mol Neurobiol 54: 5780-5797, 2017.

19. Chang N, Ahn SH, Kong DS, Lee HW and Nam DH: The role of STAT3 in glioblastoma progression through dual influences on tumor cells and the immune microenvironment. Mol Cell Endocrinol 451: 53-65, 2017.

20. Siddiquee K, Zhang S, Guida WC, Blaskovich MA, Greedy B, Lawrence HR, Yip ML, Jove R, McLaughlin MM, Lawrence NJ, et al: Selective chemical probe inhibitor of Stat3, identified through structure-based virtual screening, induces antitumor activity. Proc Natl Acad Sci USA 104: 7391-7396, 2007.

21. Zhou C, Tong Y, Wawrowsky K and Melmed S: PTTG1 acts as a STAT3 target gene for colorectal cancer cell growth and motility. Oncogene 33: 851-861, 2014.

22. Sarper SE, Inubushi T, Kurosaka H, Minagi HO, Kuremoto KI, Sakai T, Taniuchi I and Yamashiro T: Runx1-Stat3 signaling regulates the epithelial stem cells in continuously growing incisors. Sci Rep 8: 10906, 2018.

23. Segatto I, Berton S, Sonego M, Massarut S, Perin T, Piccoli E, Colombatti A, Vecchione A, Baldassarre G and Belletti B: Surgery-Induced wound response promotes stem-like and tumor-initiating features of breast cancer cells, via STAT3 signaling. Oncotarget 5: 6267-6279, 2014.

24. Su W, Guo C, Wang L, Wang Z, Yang X, Niu F, Tzou D, Yang X, Huang X, Wu J, et al: LncRNA MIR22HG abrogation inhibits proliferation and induces apoptosis in esophageal adenocarcinoma cells via activation of the STAT3/c-Myc/FAK signaling. Aging (Albany NY) 11: 4587-4596, 2019.

25. Guha P, Gardell J, Darpolor J, Cunetta M, Lima M, Miller G, Espat NJ, Junghans RP and Katz SC: STAT3 inhibition induces bax-dependent apoptosis in liver tumor myeloid-derived suppressor cells. Oncogene 38: 533-548, 2019. 
26. Chuang PY and He JC: JAK/STAT signaling in renal diseases. Kidney Int 78: 231-234, 2010.

27. Banerjee $\mathrm{K}$ and Resat $\mathrm{H}$ : Constitutive activation of STAT3 in breast cancer cells: A review. Int J Cancer 138: 2570-2578, 2016.

28. Zhi T, Jiang K, Xu X, Yu T, Zhou F, Wang Y, Liu N and Zhang J: ECT2/PSMD14/PTTG11 axis promotes the proliferation of glioma through stabilizing E2F1. Neuro Oncol 21: 462-473, 2019.

29. Luwor RB, Baradaran B, Taylor LE, Iaria J, Nheu TV, Amiry N Hovens CM, Wang B, Kaye AH and Zhu HJ: Targeting stat 3 and smad7 to restore TGF-beta cytostatic regulation of tumor cells in vitro and in vivo. Oncogene 32: 2433-2441, 2013.
30. Boelaert K, McCabe CJ, Tannahill LA, Gittoes NJ, Holder RL, Watkinson JC, Bradwell AR, Sheppard MC and Franklyn JA: Pituitary tumor transforming gene and fibroblast growth factor-2 expression: Potential prognostic indicators in differentiated thyroid cancer. J Clin Endocrinol Metab 88: 2341-2347, 2003. 